\title{
Towards Rich Motion Skills with the Lightweight Quadruped Robot Serval - a Design, Control and Experimental Study
}

\author{
Peter Eckert ${ }^{1}$, Anja E. M. Schmerbauch ${ }^{1,2}$, Tomislav Horvat ${ }^{1}$, Katja Söhnel ${ }^{3}$, Martin S. Fischer ${ }^{3}$, \\ Hartmut Witte ${ }^{2}$, and Auke J. Ijspeert ${ }^{1}$ \\ 1 Biorobotics Laboratory, École Polytechnique Fédérale de Lausanne, Switzerland \\ ${ }^{2}$ Fachgebiet Biomechatronik, Technische Universität Ilmenau, Germany \\ 3 Institut für Zoologie und Evolutionsforschung, Friedrich-Schiller-Universität Jena, Germany
}

\begin{abstract}
Bio-inspired robotic designs introducing and benefiting from morphological aspects present in animals allowed the generation of fast, robust and energy efficient locomotion. We used engineering tools and interdisciplinary knowledge transferred from biology to build low-cost robots able to achieve a certain level of versatility. Serval, a compliant quadruped robot with actuated spine and high range of motion in all joints was developed to address the question of what mechatronic complexity is needed to achieve rich motion skills. In our experiments, the robot presented a high level of versatility (number of skills) at medium speed, with a minimal control effort and, in this article, no usage of its spine. Implementing a basic kinematics-duplication from dogs, we found strengths to emphasize, weaknesses to correct and made Serval ready for future attempts to achieve more agile locomotion. In particular, we investigated the following skills: trot, bound (crouched), sidestep, turn with a radius, ascend slopes including flat ground transition, perform single and double step-downs, fall, trot over bumpy terrain, lie/sit down, and stand up.
\end{abstract}

\section{Introduction}

When we think about animals, we see many species moving dynamically in their natural habitats. While one also observes static behaviors, being in motion is characteristic for most of animal life. The variety of motion skills present in a single species is vast and more refined than what robotics researchers were able to achieve so far with technology. Two aspects that are especially striking in this context are versatility and agility. Presented originally in [1], agility is defined as follows: "Agility is representing a previously acquired and size dependent set of locomotion skills, executed in a precise, fast and ideally reflexive manner to an outside stimulus.". Comparing this definition to the one of versatility from the Oxford Dictionary (en.oxforddictionaries.com): "Ability to adapt or be adapted to many functions or activities" a new definition of agility as fast versatility or fast execution of rich motion skills may be called for. In consequence, one way to reach agility, could be to first achieve versatile behavior followed by an increase in its execution speed.

Machines with relatively simple underlying principles (e.g., cars or bikes) can move very well in our environment and navigate even through difficult terrains. In legged robotics on the other hand, whose motivation is high adaptability to uneven or difficult terrains [2], such fast and reliable locomotion is yet to be achieved. It is still unclear, although explored in many laboratories all over the world, what level of mechatronic complexity is minimally needed and sufficient to realize agile motion.

Two approaches, often described and used in control, are templates and anchors [3], both incorporating information gained from observation and analysis of animals. Hereby, a template, following the 
strictly bio-inspired direction, is simplifying the animal and its motion to the highest degree, enabling comparison on the principle-level between species. Template models can be tested against empirical data (for example the sping-loaded-inverted-pendulum-model). Anchors build upon templates and embed them in a more complex and realistic morphological and physiological model (towards bio-mimicry). Here details ranging from muscle-placement, specific joint torques up to the underlying neural control networks can and should be integrated. Usage of both, templates and anchors, in combination with detailed mechanical models and real robot hardware as 'physical simulator' to explore specific (neuro-)mechanical questions is possible. Our work uses such approaches, but in a middle way, finding an acceptable level between biological detail and complexity of implementation. This includes simple, template-like control methods with slight bio-mimicry influences, e.g., in geometry and kinematics of the robot.

This article, based on a review of the versatile behavior found in legged locomotion, will highlight our efforts in creating a small and safe experimental platform achieving the first step towards agility and rich motion skills (only a subset of implemented motions is described here, due to the scope of the article). The purpose of the robot is its usage as a system for active research, as well as an educational tool.

\section{State-of-the-Art: Versatile Legged, Terrestrial Robots}

Terrestrial legged robots range from insect scale up to large systems the size of a small horse [4]. In this short review, our focus on versatility shown by different quadrupedal robots is highlighted in the following paragraphs. Classifying existing robots by their versatility or agility in a benchmark like [1] is difficult, as only information from previous publications is available. Often included are however the absolute or relative speed, geometric measures and sometimes slope-inclinations, jump-heights or the robot's capabilities for turning. We group robots according to their mass (Group 1: $m>10 \mathrm{~kg}$, Group 2: $10 \mathrm{~kg}>\mathrm{m}$ ), presenting them on a qualitative level for their versatility and main characteristics.

In the first group, consisting of MIT-Cheetah I+II [5,6], ANYmal [7], StarlETH [8], Scout II [9] and Canid [10], the use of electrical actuation is prominent. The robots' size and weight are such that one can employ customized electric actuation with or without passive series elastic elements, e.g., high-power-density in MIT-Cheetah and highly integrated series elastic actuators in ANYmal. This group of robots is often adapted to different purposes reaching from navigation and spatial mapping in cluttered terrains, mobile manipulation to very high dynamic locomotion. The available payload allows for a high variety of sensors to be equipped and used in model-based, closed loop control schemes. Another important aspect is the possibility to handle the robot with less than two handlers, making them very well suited as sturdy experimental platforms, also for questions other than locomotion. Restrictions for morphological research is present due to the weight. Filigrane passive compliant structures, like toes, or a partially passive compliant spine are complicated to implement with current technical means, as the employable compliance can often not support the robot's mass. This scaling related effect, is most visible in MIT-Cheetah, switching from an actively bendable spine in Version I to a completely rigid trunk in Version 2. An exception, due to its weight of roughly $11 \mathrm{~kg}$, is presented with Canid. Its flexible trunk and wheg-like legs enable the robot to jump, indicating the use of high power density, but commercially standard electric actuation.

Small robots, here even under $5 \mathrm{~kg}$, represent the second and most influential group for the work presented in this paper. We selected Tekken 1 and 2 [11,12], Puppy I and II [13,14] as well as Cheetah-Cub [15], Bobcat [16], Oncilla [17], Lynx [18] and Cheetah-Cub-S [19]. For this class 
of robots a different development scheme can be employed. Additional to a general light-weight approach, very high cost-reduction becomes feasible. As the robots weight decreases, so does the necessary torque to induce movement, enabling the use of purely commercial actuation technologies down to high grade RC-servo-motors. Most robots presented, use passive elastic elements in legs or trunk to minimize active actuation and research more morphology-related aspects of locomotion. This includes the impact of the spring loaded pantographic leg-structures inspired by [20] and control methods (like CPGs) not relying on precise sensor feedback or torque-control. Nevertheless, as shown already in Tekken 1 and 2, stable locomotion, even on relatively rough terrain remains possible. This ability is refined by adding feedback in form of different reflexes to adapt and react to the environment, as in the Tekken robots and Oncilla [2]. Losing the capability to carry heavy sensory equipment, e.g. LIDAR (needed for quick and precise spatial mapping) is compensated with an ease of use as experimental platforms for template research, e.g. the application of flexible spines for steering and improved locomotion (Cheetah-Cub-S, Lynx). Small robots can be handled safely by a single operator, even after very basic training. Cost-efficient production allows for groups without high budget to copy, maintain and use small robots as physical simulators and thus increases options to verify theoretical or simulation work.

The presented state-of-the-art clarified for us two main aspects: (a) Visible from our qualitative analysis we can state that researching versatility and often agility is possible with any size of quadrupedal robot. The distinction has to be made if one desires an all-round robot (group 1) or a more specialized system (group 2, besides Oncilla). (b) Our intent to use robots as educational and research platforms, implies safety and ease of use to be of the essence. This is generally prominent group 2. For Serval, we aim for a middle way in between both groups.

\section{Robot Development}

Animals, despite their enormous skill to balance, often trip, fall or run into their surroundings. To compensate for the lack of environmental sensing and reflexive mechanisms, our robot needed at least a sturdiness somewhat close to the animal one. Compliant elements in key positions in combination with a sturdy, but very lightweight skeleton should thus build Serval's mechanical core, also resulting in a low inertia system. Relatively high power-density actuation and processing power enable fast execution of motion commands. If these measures fail to enhance the movement and protect our robot, the modular design approach enables easy and quick repairs. Following our goal of reaching versatile movement, our robot control needed to consist of a flexible and modular approach. Formed around an open-loop CPG-controller for basic movement generation, we implemented different behaviors, which cohere with our vision of versatility and can potentially be executed quickly to achieve agile movement. If these aspects work together symbiotically within a compliant and relatively powerful mechatronic design, we are confident to be capable of a good grade of versatility or even agility.

\subsection{Mechanical Development}

Mechanical development in Serval presents a combination of tested mechanisms from previous robots with the goal of enhancing their advantages while canceling out as many disadvantages as possible. The resulting robot consisted of a modular design built around Dynamixel MX64R/MX28R servo motors and an Odroid XU4 SBC. One can distinguish three reusable main units: (1) trunk, (2) leg and (3) spine unit, illustrated in Figure 1. The trunk-unit acts mainly as a connection hub, and 
housing for the legs, spine as well as electronics. Due to the scope of the paper, we will refrain from describing it in detail. The units are integrated and extended with a new foot design as well as in-series elastics for motor and mechanics protection from impacts. To reach a lightweight as possible design and with ease of implementation in mind, we built the robot's skeleton from lightweight aluminum ( $\mathrm{Al}$ ), steel (only for axes) and POM (engineering plastic), machined with classical CNCmilling, CNC-Laser, and bending techniques. The leg-unit incorporated at its base an ASLP-leg (advanced spring-loaded pantograph) in the design of the previously developed Cheetah-Cub-AL (go.epfl.ch/CheetahCubAL), see Figure 2. Due to the size of the robot and thus the legs' dimensions and resulting lever arms, relatively stiff springs had to be integrated. Please find a 3D-PDF of Serval here: go.epfl.ch/3DPDFServal.

Leg-unit: ASLP segmentation (fore and hind differ) was kept as a scaled version of CheetahCub-AL to re-use as much of the previous design as possible. Additionally, a passive-compliant carpal-joint (wrist joint) was added to the fore legs, to test the possibility of small-step-ups without sensory feedback (future work). The leg unit was iterated once, as the diagonal spring-mechanism caused failure of blocking the leg due to quick wear and tear of its rectangular guidance (due to manufacturing imprecision and choice of material). After exchanging it with precisely turned AL-inner- and POM-outer-guide, this issue was solved. The legs' springs work as an antagonist to their respective motor, thus enabling relatively fast extension with a force that can be deduced from the springs' prior deflection. Although this fast extension might lead towards explosive movements in the future, a trade-off had to be chosen in the current design. For motions like jumping, high forces are needed, thus depicting the need for very high spring-stiffness. This would exceeding the resulting compression capabilities of the servo-motors employed in our work and limit the adaptability of the robot to uneven terrains of falls due to a lack of energy absorption/dissipation in the legs. Jumping would thus only be possible with a different actuator choice or a supplementary leg extension mechanism. Between robot adduction/abduction (AA), with direct actuation located roughly on the hip axis height, an in-series elastic was mounted. The very stiff mechanism, still used in the experiments presented in this paper, was later-on exchanged for a torsion tube-like structure, made from NiTinol-wires in its super-elastic state. Flexion of the leg due to external forces resulted in a torsional displacement, reducing direct impact propagation to the AA-actuation. This mechanism could be combined with a rotational damper to dissipate impact energy instead of just smoothing the peak forces. After testing different designs, we decided to include a segmented, spring-loaded foot with two rounded, claw-shaped toes. We hypothesized the need for ground adaptation due to the large AA-capability of the robot (changing lateral angle to the ground) and expected better grip on rough or granular terrains.

Spine-unit: Three active DOF, one for rotation in the transversal and two for rotation in the saggital plane, were forming the core of the spine including a small handle and IMU-connector on the middle motor. The elements were connected through bent AL-pieces and leaf-springs made of four NiTinol wires in parallel. The hind elastics were connected in a cross shape, stiffening the spine in one direction and enabling compliant behavior in the orthogonal other to comply with the in-series load for their respective motor. The overall arrangement of the springs is approximating a classical rectangular leaf spring. This results in different overall behavior of the springs depending on the direction of the applied forces, inducing small displacement in one and larger in the orthogonal direction. For adjustment of the allowable deflection and stiffness of the springs, clamps can be added to the spring-fixations, shortening the free length of the mechanism. Stiffness is also reducable by removing wires from the set. For our spine, we desire relatively stiff connections enabling the direct transmission of forces in the steering direction and softer springs for saggital movement. 

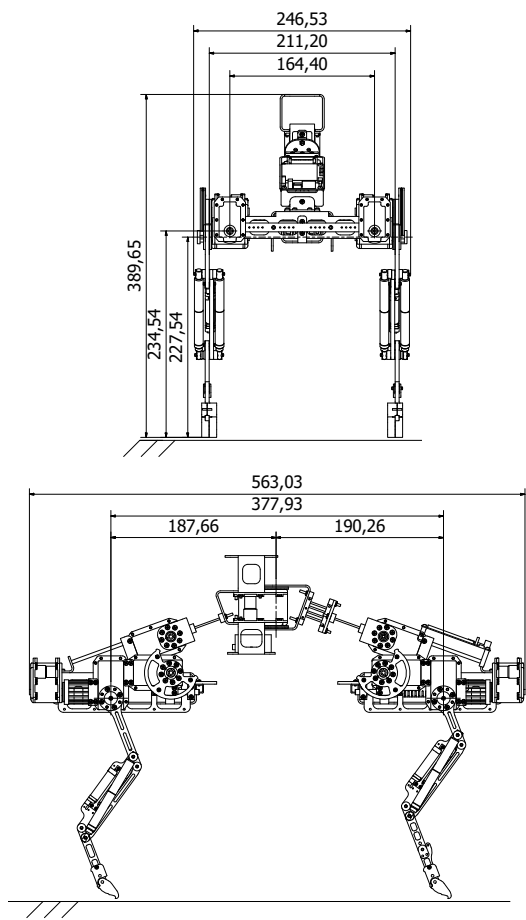

Fig. 1: Front and side view of Serval with characteristic measurements, abstracted CAD.
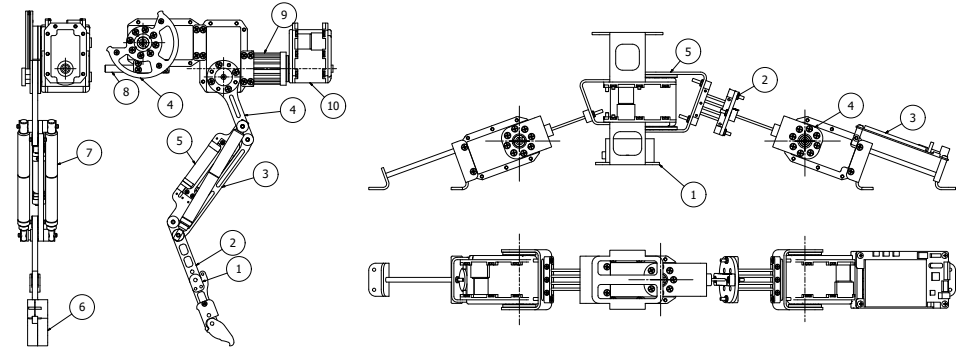

Fig. 2: (left) Serval's leg unit from a side and front view: (1) carpal joint, (2) 13-segment (parallel to 11), (3) 12-segment, (4) 11-segment (parallel to 13), (5) parallel spring (uncompressed), (6) compliant foot (2 toes), (7) diagonal springs (symmetric to saggital plane of the leg), (8) rotary fixation for the leg-unit, (9) AA-in-series-elastic, (10) AA-motor with axis slightly displace from hip axis, higher ground-clearance when moving to the outside, smaller to the inside; (right) Serval's spine unit from a side and top view: (1) IMU fixation, (2) Cross-joint with Nitinol-leafsprings, (3) screen for basic HMI, (4) up-down DOF, (5) steering DOF.

We believe that a certain amount of compliance in the spine is necessary when impacts are too large to be absorbed by the legs alone and is yet to be tested. Usage of NiTinol as shape memory 
allow with resulting stiffness changes was omitted due slow reaction time (heating/cooling) and high unpredictability in its transition states.

\subsection{Control Development}

The first implementation towards versatile movement with Serval consisted of replaying modified kinematic data from dogs [21]. This approach, due to the readiness-state of the robot hardware and control (no sensors integrated at the time, no reflexes), was performed in open-loop and is thus a basic control to be extended in current and future work.

Analysis and mathematical representation of motion capture (MOCAP) data from trained Border Collies enabled the foot trajectory generation for Serval's inverse kinematics control. Four dogs' data was available to be processed to obtain kinematic data of different gaits. Figure 3 shows an example for fore- and hind-foot trajectory, illustrating differences in, e.g., vertical displacement as well as distances to hip joint axes. All Border Collies were taller than $45 \mathrm{~cm}$ at withers resulting in the need for scaling of established trajectories to the robot's size. We fitted four cubic Bézier curves to the data, to mathematically recreate the complex shape of a real animal foot-locus. Junction Points were positioned vertically to the hip axis and on the transition from stance to swing and swing to stance phase. Consequently, the calculation of the inner Bézier points completed the definition the dog foot-loci with cubic Bézier curves. We proposed a parametrization approach using hip height $(\mathrm{H})$ as origin $\left(\mathrm{x}_{0}, \mathrm{z}_{0}\right)$, step height $(\mathrm{h})$, compression factor $(\mathrm{c})$ included in ( $\left.\mathrm{h}=(1-\mathrm{c}) \mathrm{h}\right)$, step length (SL) and length proportion per direction (LR and LL) for trajectory modification in experiments, see Figure 3. The use of take-off and touchdown angles, like in Cheetah-Cub-AL's foot-locus parametrization [22], was deliberately omitted to keep the ratios and proportions of foot trajectories imported from animal data intact but retain the possibility for adjustment of the general trajectory size. Having extracted the foot-loci from cubic Bézier curve interpolation (and depending on the leg timing), the data was ready to be "replayed" by Serval. Underlying control was using an adapted framework from Pleurobot [23]. On a high-level, we used the controller's state machine along with a CPG-network to update the foot-position constantly, generating our different motions. Duty factor was not commanded explicitly but depended on the implemented trajectory. The combination of the trajectory with correct timing of the inter-limb coordination let to a specific gait.

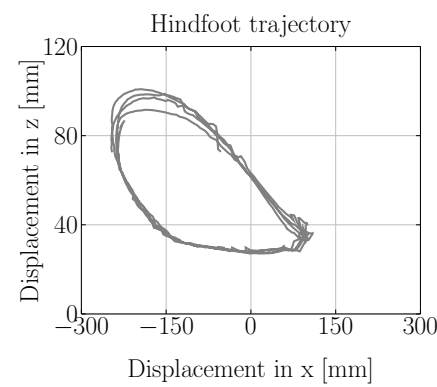

(a) HL-Ethan

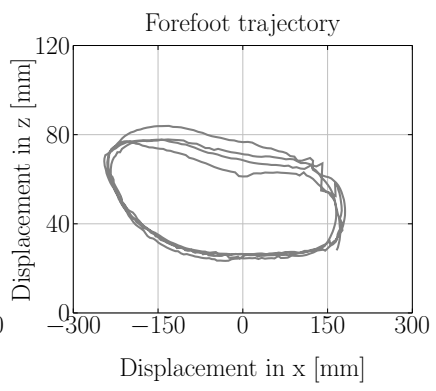

(b) FL-Ethan

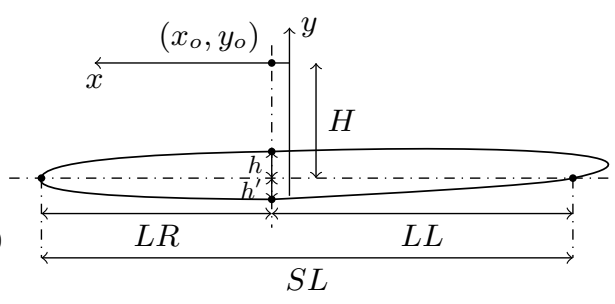

(c) Parametrization

Fig. 3: 3a and 3b Forefoot and hindfoot trajectory for trot of a Border Collie (Ethan); head to the right; rather flat elliptic shape in the fore and angled elliptic shape in the hind (more ground clearance); 3c Parametrization method for experimental implementation. 


\section{Experimental Validation}

Most of the here presented experiments do not yet employ active trunk movement. For debugging purposes, we decided to block the spine with two POM plates and free it only for use in turning maneuvers. All tests presented here were done tethered for off-board power supply.

After preliminary adaptation of the scaling to match our dog-data to the robot's geometry, we focused on different skills to test robot mechanics and its suitability for versatile and agile locomotion. A subset is presented in this article: trot (with and without AA), bound (crouched), sidestepping, turning with a radius, slope-up with a flat ground transition, single and double step-down, fall absorption, rough terrain, lying/sitting down, and standing up (Videos: go.epfl.ch/ExperimentsServal ${ }^{4}$ ).

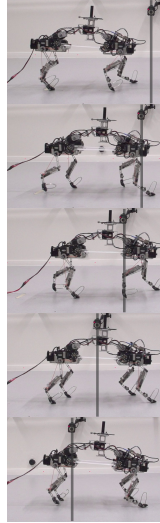

(a)

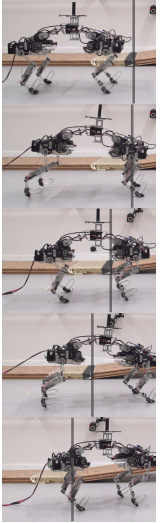

(b)

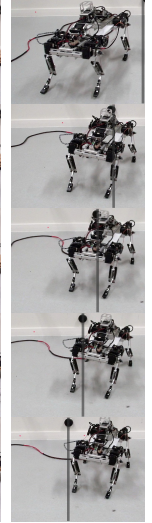

(c)

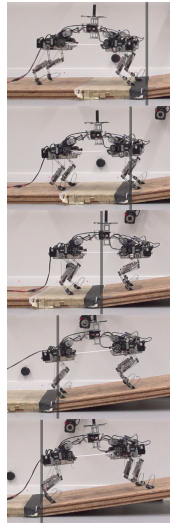

(d)

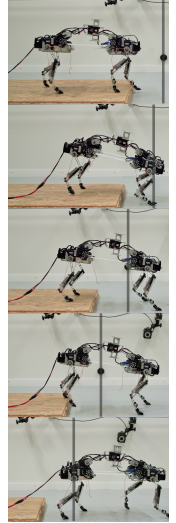

(e)

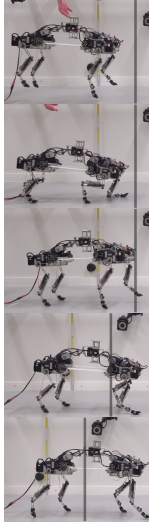

(f)

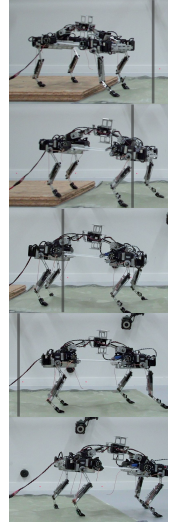

(g)

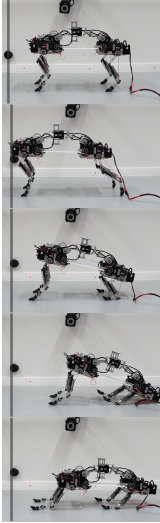

(h)

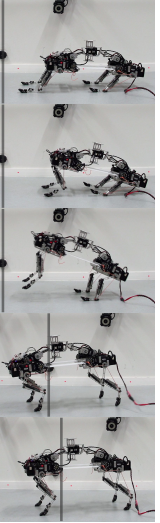

(i)

Fig. 4: Snapshots of Serval (one skill per column, start on the top, transparent bar gives a fixed reference between images: 4a trot (dynamic movement, sliding at touch-down and toe-off, decreasing efficiency, speed at $0.83 \mathrm{~m} / \mathrm{s}$ ), $4 \mathrm{~b}$ crouched bound (dynamic movement, left-right-symmetry, sliding prominent at toe-off, small turn to the left), 4c side-step (lateral combined with backwards motion, stick-slip and stuttering due to foot-geometry and small ground clearance during swing), $4 \mathrm{~d}$ bounding upslope $\left(16^{\circ}\right.$ inclination $(\approx 28.7 \%)$, transition from flat to inclined terrain, heading relatively straight), $4 \mathrm{e}$ trotting down a single step $(63 \mathrm{~mm}(\approx 30 \%$ of leg length), success-rate of $70 \%$, shock absorption through the parallel spring), $4 \mathrm{f}$ falling (height $\approx 70 \%$ of leg length while trotting, success rate $>90 \%$, complete flexion of the parallel spring and touch down of the knee, passive flexion of the diagonal springs), $4 \mathrm{~g}$ trotting on a smoothed bumpy terrain (after initial step-down, deviations from straight path and uncontrolled movements are present), $4 \mathrm{~h}$ and $4 \mathrm{i}$ lying down and standing up (fully hard-coded motion-sequence inspired by dogs).

\subsection{Flat Terrain Locomotion}

Trot: Following the gaits often observed in animals, the running trot was tested $(h=0.03 \mathrm{~m}$, $S L=0.15 \mathrm{~m}$ and $F=1.5 \mathrm{~Hz}$ ), see Figure 4 for snapshots. We used two different settings for the AA. When moving on flat ground, the hind legs were flexed towards the sagittal plane and forelegs extended in the opposite direction. This posture is observed in dogs when moving in medium to

\footnotetext{
4 no sound available due to recording without microphone
} 
high speeds to possibly enable overlapping of their feet during motion. This way, the hind legs can provide most of the propulsion whereas fore legs stabilize the robot. For other tasks, like step-downs or backward trot, we set the AA straight. This decreased variation in the robot's roll-angle when perturbed and was thus useful in cases where self-stabilization was the top priority. In Figure 5 we present an example of GRFs (Kistler, type 9260AA3, mounted side-by-side (left-right) and covered with non-reflective tape within a catwalk) that mainly confirms the visual impression of characteristic trotting and bounding from Figure 4. The main challenge in obtaining reliable GRF-data was the feed-forward generation of the robot gait. The robot had to run straight over the two force-plates, allowing the distinction between left and right feet and thus the generation of valid GRF-gait-data. As the robot was often reacting to changing surface conditions by changes in direction, separated touchdowns were not impossible, but difficult to achieve. This increased the number of experimental runs that had to be performed to acquire clean GRF-data. The robot showed the main characteristics of a trot-pattern repetitively, see Figure 4a.
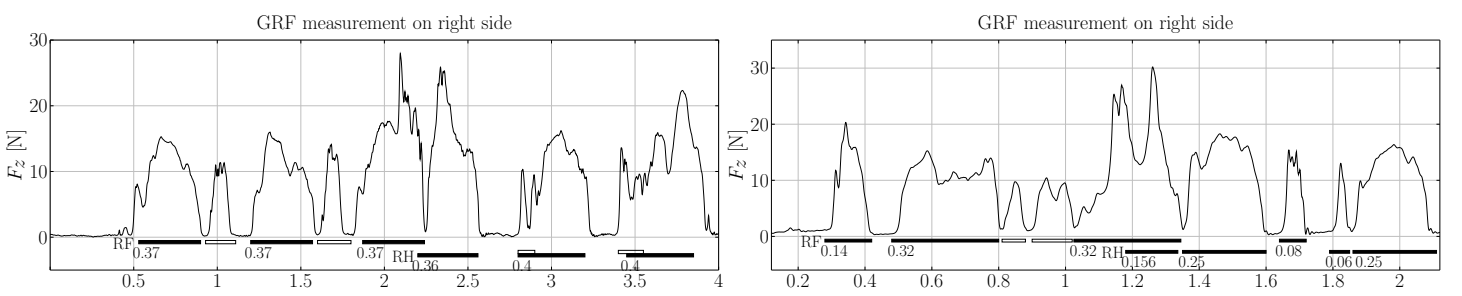

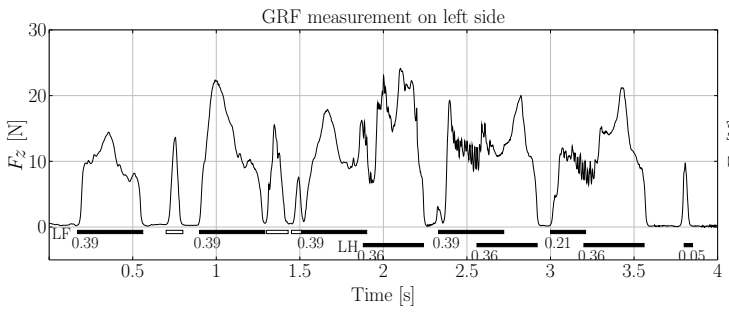

(a) trot

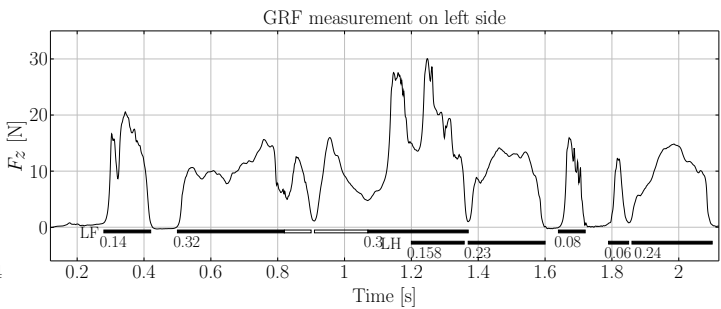

(b) bound

Fig. 5: 5a GRF measurements for a trot gait, footfall patterns indicated in black with expected characteristic appearance, foot sliding is represented by white boxes, and individual duty factors are marked, mean duty factors: $D F_{L F}=0.36, D F_{L H}=0.37 D F_{R F}=0.37, D F_{R H}=0.39$ and $D F_{a v}=0.37$; GRF patterns are similar to Cheetah-Cub, with never the full robot weight $(33 N$, without battery) on one single foot; Peaks are visible, e.g., at $1 s$ on the left fore foot that was caused by the hind foot obtaining high traction leading to higher compensation forces in the diagonally opposite foot; 5b GRF measurements for a bounding gait using a crouched posture; mean duty factors: $D F_{L F}=0.38, D F_{L H}=0.34 D F_{R F}=0.39, D F_{R H}=0.36$ and $D F_{a v}=0.37$; robot weight is evenly distributed on two sagitally opposite feet (left-right-symmetry); the peak at $\approx 1.3 \mathrm{~s}$ is an example of all feet touching the ground at the same time, resulting in application of the robot's full weight as vertical force.

The reduced controllability of the ASLP leg due to under-actuation can explain the phenomenon of occurring slippage to compensate early touch down of the feet, already observed in Oncilla and Cheetah-Cub $[15,17,2]$. Further, as we were using a gait not tailored specifically to the robot but 
stemming from kinematic recordings, a mismatch is possible. From a mechanics point of view, we see future need for new materials with anisotropic friction to enhance propulsion in one and allow for slippage in the other direction. Nevertheless, the trot gait was very stable, out-of-the-box and enabled the robot to locomote at a speed of $0.83 \mathrm{~m} / \mathrm{s}(\mathrm{FR}=0.32)$ with a visible trot footfall pattern, see Figure 5a.

Bound (crouched): The crouched bound (Figure 4b), is used by cats to climb very steep slopes over $50 \%$ inclination [24], but is also a useful gait when testing active spine movement $[16,18]$. For us, as we did not yet free the spine, the crouched bound was used mainly for slopes, as described later. Nevertheless, a feasible gait was also obtained when running on flat terrain. Walking foot-loci with changed inter-limb timing were used to achieve the motion. Bounding showed good directional stability, visible from Figure 4 and an almost perfect representation of the desired footfall pattern, illustrated in Figure 5b. At seldom occasions stick-slip is visible. We hypothesize that improvement towards a non-slip gait is possible when freeing the spine and improving ground contact with anisotropic friction material on the feet.

Side-stepping: We included lateral side-stepping in our initial experiments as preparation for later execution of a lateral stepping reflex [2]. A spatial 8-figures was commanded to the robot's feet, hence using the AA to push the robot to one side and shifting body weight away from the side whose feet should be in swing-phase. Without touching the ground, Serval was able to perform the task, but as soon as in contact, stick-slip with the feet's hard edges due to little ground clearance made a movement impossible. The snapshots in Figure 4 demonstrate an alternative, artificial gait using AA, allowing for lateral-aft motion. A pure lateral movement might be difficult to achieve, if posture is not kept balanced through a posture-adaptation-reflex, allowing the swing legs to execute their movement without touching the ground.

Turning with a Radius: The last movement on flat ground essential for a versatile system is the ability to turn. Here we used a combination of asymmetric stride length [17] and lateral spine-deflection. The resulting turn was again rather perturbed by slippage but reaching a small turning-radius of $0.58 \mathrm{~m}$. Adding the AA-movement to the two previously mentioned strategies should reduce slippage and make turning more repeatable.

\subsection{Inclined Surfaces, Perturbations, Stability, and Rest-Position}

Slope-up: Experiments have been performed to identify the maximum slope Serval can climb up, using a bound and crouched posture adaptation, see Figure 4d. The maximum inclination feasible with an open-loop gait amounted to $20^{\circ}(\approx 36.4 \%)$ with a transition from flat ground to the slope. Without heading-correction, substantial slippage in the propulsion phase of the toe-off and drift to the side could be observed. Serval could repeatedly move on a $16^{\circ}(\approx 28.7 \%)$ slope with little drift. Smaller inclinations could also be achieved with a trot-gait. Locomotion down-slope was also possible. We did no test lateral inclinations, as an appropriate test setup was not available at the time.

Single and double Step-down We included step-down experiments to demonstrate the selfstabilizing behavior of the robot and the gait robustness, see Figure 4e. The goal was the determination of the maximum step height which the robot can go down in open-loop while reliably using its legs' compliance. The applied gait was an unmodified trot. The requirements for a successful try was the continuation of a stable gait for at least $2 m$ after step-down. We performed at least ten runs per step-height. With reliability of $100 \%$ Serval adapted to step-downs of $53 \mathrm{~mm}$ that amounts to $\approx 25.2 \%$ of its leg length. The largest step of $63 \mathrm{~mm}(\approx 30 \%$ of leg length) resulted in a success-rate 
of $70 \%$. Step downs over two consecutive steps were successfully performed in $90 \%$ of the cases, at the height of $26 \mathrm{~mm}(\approx 12.5 \%$ of leg length).

Fall Absorption: Robustness is key, as versatile and agile motion can lead to falls and failures rather quickly. Dropping the robot from a maximum height of $70 \%$ leg length while running with a trot showed that it was possible to overcome impacts and continue locomotion in this idealized scenario, see Figure 4f. In the first images after touch down one notices the strong deflection of all leg-springs and the resulting push-off. A critical point is presented by the touch-down of the leg's middle segment and full compression of the parallel spring already for this drop height. However, the robot was always able to regain a stable trot after some stepping cycles. This result is encouraging, as it shows robustness as long as the force is transmitted in a way that the leg-compliance can disperse the impact. Additional tests from other angles and heights should be performed to characterize the robot further and test the new in-series implementation in AA and spine joints.

Rough Terrain: Moving over sharp vertical obstacles, using only open-loop, was already found to be widely impossible in our experiments with Oncilla [2]. As an additional test of Serval's stability, we decided to let it run on a smooth, but bumpy terrain after a small step-down, see Figure $4 \mathrm{~g}$. Without controlling the heading, the robot was sliding to different sides, moving backward, but in the end, finishing the distance over the plate. This highly irregular behavior is of limited use in real scenarios, but again, underlines the robot's stability due to its compliance. Heading and posture control may build on this stability to enable new application environments and increase the robot's real-world capacity and adaptation.

Lying/Sitting down and Standing-up: We defined the transition from sitting/lying to a normal standing posture as a part of versatility. Consequently, these behaviors were implemented and tested. Kinematic data for the robot joints was extracted from MOCAP of Border Collies, and the movements were implemented with success, as hard-coded motion-sequences [25], see Figure 4h, 4i.

\section{Conclusion and Discussion}

When comparing to our reviewed state-of-the-art, Serval is positioning itself as a versatile robot with a high level of mobility at medium speeds. Robots showing comparable skills are Oncilla, Tekken 2 and Scout II. ANYmal, StarlETH, Canid, as well as the MIT Cheetahs are still ahead regarding performance (through higher grade actuation and closed-loop control) or simply present a different set of skills (such as walking stairs or jumping). With the number of successfully implemented skills, using a basic kinematics-duplication, we debugged the robot hardware, found out strengths to emphasize (compliance and adaptable feet), and weaknesses to correct (friction of ground contact and low stiffness of spine/AA). All in all the initial tests shown here were a great success generating valuable insights towards hard- and software development.

Our study of flat terrain locomotion demonstrated Serval's ability to achieve many motion patterns, simply by replaying parameterized dog-foot-loci. Further investigation of optimal gaits tailored to the robot should improve the existing patterns. Further work should investigate if bio-inspired, but artificially generated trajectories, like in the Cheetah-Cub-Family and Oncilla, have an advantage over replaying kinematic data from example animals. The robot's capability of ascending slopes in open-loop is very promising. It already improved markedly from max $15^{\circ}$ in Oncilla (closed-loop) to max $20^{\circ}$ in Serval. In further work and with PAD as well as better surface friction included, even steeper slopes will be feasible. Regarding stepping down, Serval showed remarkable results and thus followed up on the success in Cheetah-Cub, even increasing the percentile maximal step height by $10 \%$ and success-ratio by $50 \%$ in this direct comparison [15]. The next logical step is to increase 
maneuverability by implementing step-ups through reflexes. Both rough terrain locomotion as well as fall absorption were handled repeatedly well. Aforementioned skill is a valuable proof-of-concept of robot-robustness and highlights the importance of passive compliance in small quadrupedal robots. This passive adaptability is providing an important fail-safe if more sophisticated control (to be implemented in the future) might fail. Both motions, sitting/lying down and standing up, were achieved in ideal conditions, on flat ground without any inclinations of the robot body or even lying on the back. The robot was able to repeatedly move from one posture to the other and start trotting

afterward. To further enhance the motion-sequences, especially when not in an ideal position (e.g., on the side), further sensorization with an IMU as well as an active spine are needed.

Conclusively, Serval has the potential for agile locomotion by showing versatility within a basic setup. Relatively stiff legs allow for good shock absorption in a higher weight-range and possibly fast extension (explosive behavior) in low load cases. Modular design and adaptability of springstiffnesses enable experimental tuning for our agility tasks efficiently. Turning and locomotion in difficult terrain are in principle possible via different strategies, leveraging the high ROM in AA, spine, and legs. To enhance overall performance and protect the robot from failure, reflexive mechanisms as in Oncilla, based on appropriate sensory feedback (e.g. GRF and body inertia/heading), should extend the robot control. Additionally, to complete the level of mechatronic complexity needed for versatility and agility, we believe that freeing the active compliant spine and integrating an appropriate controller is advantageous. Investigating anisotropic friction, geometry, and stiffness of the feet to allow defined and efficient propulsion should be addressed as well.

Acknowledgements: We thank the 'Bewegungslabor (OpenLab) der westfälischen Wilhelms Universität Münster", especially Dr. Marc de Lussanet and Prof. Dr. Heiko Wagner. We wish to thank the owners of the dogs for providing the experimental subjects. This collaborative work was financially supported by the NCCR Robotics and gkf Gesellschaft für kynologische Forschung. We thank the editors and reviewers for their constructive criticism.

\section{References}

1. Eckert, P., Ijspeert, A.J.: Benchmarking Agility For Multi-legged Terrestrial Robots. IEEE Transactions on Robotics - in progress (2018)

2. Ajallooeian, M.: Pattern Generation for Rough Terrain Locomotion with Quadrupedal Robots. PhD thesis, EPFL (2015)

3. Full, R.J., Koditschek, D.E., Full, R.J.: Templates and anchors: neuromechanical hypotheses of legged locomotion on land. The Journal of Experimental Biology 2(12) (1999) 3-125

4. Ijspeert, A.J.: Biorobotics: Using robots to emulate and investigate agile locomotion. Science 346(6206) (2014) 196-203

5. Seok, S., Wang, A., Chuah, M.Y., Otten, D., Lang, J., Kim, S.: Design principles for highly efficient quadrupeds and implementation on the MIT Cheetah robot. In: Proceedings - IEEE International Conference on Robotics and Automation, IEEE (may 2013) 3307-3312

6. Park, H.W., Park, S., Kim, S.: Variable-speed quadrupedal bounding using impulse planning: Untethered high-speed 3D Running of MIT Cheetah 2. Proceedings - IEEE International Conference on Robotics and Automation 2015-June(June) (may 2015) 5163-5170

7. Hutter, M.: ANYmal - A Highly Mobile and Dynamic Quadrupedal Robot. In: Arbeitsberichte Verkehrsund Raumplanung, IVT , ETH Zurich. Volume 544. (2009) 1 - 25 
8. Hutter, M., Gehring, C., Höpflinger, M.A., Blösch, M., Siegwart, R.: Toward combining speed, efficiency, versatility, and robustness in an autonomous quadruped. IEEE Transactions on Robotics 30(6) (2014) $1427-1440$

9. Poulakakis, I., Smith, J.A., Buehler, M.: Modeling and experiments of untethered quadrupedal running with a bounding gait: The scout II robot. International Journal of Robotics Research 24(4) (apr 2005) 239-256

10. Pusey, J.L., Duperret, J.M., Haynes, G.C., Knopf, R., Koditschek, D.E.: Free-standing leaping experiments with a power-autonomous elastic-spined quadruped. SPIE Defense, Security, and Sensing 8741 (2013) 87410W

11. Kimura, H., Fukuoka, Y., Cohen, A.H.: Adaptive dynamic walking of a quadruped robot on natural ground based on biological concepts. International Journal of Robotics Research 26(5) (2007) 475-490

12. Fukuoka, Y., Kimura, H.: Dynamic locomotion of a biomorphic quadruped Tekken robot using various gaits: walk, trot, free-gait and bound. Applied Bionics and Biomechanics 6(1) (2009) 63-71

13. Iida, F., Pfeifer, R.: Cheap rapid locomotion of a quadruped robot: Self-stabilization of bounding gait. In: Proceedings of the 8th International Conference on Intelligent Autonomous Systems (IAS-8). Volume 8. (2004) 642-649

14. Iida, F., Gómez, G., Pfeifer, R.: Exploiting body dynamics for controlling a running quadruped robot. In: 2005 International Conference on Advanced Robotics, ICAR '05, Proceedings. Volume 2005., IEEE (2005) 229-235

15. Spröwitz, A., Tuleu, A., Vespignani, M., Ajallooeian, M., Badri, E., Ijspeert, A.J.: Towards dynamic trot gait locomotion: Design, control, and experiments with Cheetah-cub, a compliant quadruped robot. International Journal of Robotics Research 32(8) (jul 2013) 932-950

16. Khoramshahi, M., Sprowitz, A., Tuleu, A., Ahmadabadi, M.N., Ijspeert, A.J.: Benefits of an active spine supported bounding locomotion with a small compliant quadruped robot. In: Proceedings - IEEE International Conference on Robotics and Automation. (2013) 3329-3334

17. Sprowitz, A.T., Tuleu, A., Ajaoolleian, M., Vespignani, M., Moeckel, R., Eckert, P., D'Haene, M., Degrave, J., Nordmann, A., Schrauwen, B., Steil, J., Ijspeert, A.: Oncilla robot: a versatile open-source quadruped research robot with compliant pantograph legs. Frontiers in Robotics and AI 5 (2018) 67

18. Eckert, P., Sprowitz, A., Witte, H., Ijspeert, A.J.: Comparing the effect of different spine and leg designs for a small bounding quadruped robot. In: Proceedings - IEEE International Conference on Robotics and Automation. Volume 2015-June. (2015) 3128-3133

19. Weinmeister, K., Eckert, P., Witte, H., Ijspeert, A.J.: Cheetah-cub-S: Steering of a quadruped robot using trunk motion. In: 2015 IEEE International Symposium on Safety, Security, and Rescue Robotics (SSRR). (2015) 1-6

20. Witte, H., Hackert, R., Lilje, K.E., Schilling, N., Voges, D., Klauer, G., Ilg, W., Albiez, J., Seyfarth, A., Germann, D., Hiller, M., Dillmann, R., Fischer, M.S.: Transfer of biological principles into the construction of quadruped walking machines. Proceedings of the 2nd International Workshop on Robot Motion and Control, RoMoCo 2001 (2001) 245-249

21. Söhnel, K., Andrada, E., De Lussanet, M.H.E., Wagner, H., Fischer, M.S.: Kinetics in Jumping Regarding Agility Dogs. (2017)

22. Tuleu, A.: Hardware, software and control design considerations towards low-cost compliant quadruped robots. PhD thesis, EPFL (2016)

23. Horvat, T., Karakasiliotis, K., Melo, K., Fleury, L., Thandiackal, R., Ijspeert, A.J.: Inverse kinematics and reflex based controller for body-limb coordination of a salamander-like robot walking on uneven terrain. In: IEEE International Conference on Intelligent Robots and Systems. Volume 2015-Decem., IEEE (sep 2015) 195-201

24. Smith, J.L., Carlson-Kuhta, P., Trank, T.V.: Forms of forward quadrupedal locomotion. III. A comparison of posture, hindlimb kinematics, and motor patterns for downslope and level walking. Journal of neurophysiology 79(4) (oct 1998) 1702-1716

25. Schmerbauch, A.E.M., Eckert, P., Witte, H., Ijspeert, A.J.: Implementation and analysis of rich locomotion behavior on the bio-inspired, quadruped robot Serval (2017) 